\title{
Diabetes mellitus and treatment: a study of social representations
}

\begin{abstract}
Background: This study aimed to apprehend the social representations elaborated by people who have diabetes about the disease and the treatment. As many as 31 people, with ages ranging from 34 to 76 years old $(\mathrm{M}=55.68, \mathrm{SD}=11.6)$, who responded to the questionnaire are heat-demographic and depth interview. The data were submitted to the Alceste software and analyzed using descriptive statistics and lexical analysis. The results showed that the social representations were focused on the ignorance of diabetes, highlighting the surprise of diagnosis, their representations, were also anchored in nutritional and emotional factors which were permeated by negative emotions. Participants who have insulin used ratified responsibilities notions, directed to Consequences disease, such as the case of the limb amputations Those who did not used the described insulin disease linked to the triad of treatment. The results showed the importance of an interdisciplinary approach that focuses on the psychosociological support aimed at developing strategies that can be adopted to control and diabetes care.
\end{abstract}

Keywords: diabetes mellitus, social representation, social psychology
Volume 3 Issue 4 - 2018

\section{Fabrycianne Gonçalves Costa, Maria da Penha de Lima Coutinho \\ Department of Psychology, Higher Education Institute of} Paraiba, Brazil

Correspondence: Fabrycianne Gonçalves Costa, Department of Psychology, Higher Education Institute of Paraíba, Ful addressing, Rodovia BR 230, Brazil, Tel +55 999675452, Email fabrycianne@gmail.com

Received: April 02, 2018| Published: August 01, 2018

\section{Introduction}

Diabetes mellitus (DM) is a major problem of public health at the expense of its wide prevalence and its psychological impact which may be related to severe complications that compromise productivity quality of life and survival of s people and involve high costs in their treatment and complications. This is a non-transmissible chronic diseases caused by the hereditary and environmental factors, diabetes is orredecnt of absolute or relative deficiency in the secretion and/or insulin action casionando the ACU module of glucose (sugar). ${ }^{1}$

According to the American Diabetes Association (ADA) the acceptable level of glucose in the blood is up to $100 \mathrm{mg} / \mathrm{dL}$; above and the person may have hypo- or hyperglycaemic (ADA, 2015) crises. ${ }^{2}$ the disease arises and progresses insidiously from a dysfunctional impairment of the pancreas. It comprises a heterogeneous group of metabolic diseases associated with complications, dysfunction and failure of various organs, which gradually reach the vision, kidneys, brain and heart. ${ }^{3}$

The most obvious symptoms of diabetes are: too much thirs (polydipsia) excessive urine (polyuria) too much hunger (polyphagia) and weight loss. Other symptoms are: drowsiness, generalized pains, tingling and numbness, pain in the legs, cramps, nervousness, restlessness, dismay, blurred vision, physical and mental fatigue. ${ }^{3}$

According to research, the incidence and prevalence of diabetes tends to increase due to population aging, urbanization, economic development and therapeutic advances in the treatment of the disease, but especially to the current lifestyle, characterized by physical inactivity and eating habits that predispose to accumulation of body fat. ${ }^{4,5}$

According to the most recent estimates of the International Diabetes Federation (IDF), currently approximately 415 million people worldwide have diabetes, a factor that is related to 5.1 million deaths in $2012 .{ }^{6}$ In Brazil, there are about 1.4 million carriers of the disease and 500 new cases emerge every day. According to data of the Brazilian Society of Endocrinology and Metabolism-Regional Paraíba (SBEM-CP) appreciates that there are 135,000 diabetics in Paraiba scenario, $d$ these, 37,500 are located in João Pessoa. D iante the high prevalence of diabetes the World Health Organization (WHO) considers that the disease will be the seventh leading cause of death in 2030 (IDF, 2013). ${ }^{6}$

It is known that living with diabetes requires a complex adjustment in lifestyle, which involves the incorporation of therapeutic practices, changes in eating patterns, glycemic control, medication, physical activity, blood pressure maintenance and continuous follow-up of the multidisciplinary health team, aiming at a healthy life, preventing the possible acute and chronic complications.?

In this sense, the way people perceive their living conditions influence on the general control of their health / disease, making it important to understand how people with diabetes symbolize the experience produced by this reality, u ma time understand the construction. ${ }^{8}$ In the present study, it is important to note that this type of thinking can contribute to a greater visibility for this phenomenon and, consequently, to guide diabetics towards the disease and its therapeutics.

Given these assumptions, the aim-to identifies the social representations made by people who have diabetes about the disease and treatment. For the reasoning of this study we used The Teoria of R s Social representations (TRS). Social representations should be seen as a specific way of understanding and communicating what we already know. They occupy, in effect, a curious position, somewhere between concepts, whose aim is to abstract sense of the world and to introduce order and perceptions into it reproducing the world in a meaningful way. ${ }^{9}$

The genesis of the construction of social representations is anchored in two processes: anchoring and objectification, which are at the same time of a social and cognitive nature, allowing the transformation of 
what is unfamiliar into something familiar and known. ${ }^{9}$ Anchoring directs memory inward, searching for things, events and people, which it recognizes as a prototype, or recognizes itself by naming it, by comparing and interpretation. In turn, the process of objectification reproduces an unknown concept of reality, transferring it to a visible and tangible level; it is a question of how the constituent elements of representation are organized and of the way in which these elements acquire materiality and become expressions of a reality thought to be natural. ${ }^{8}$

The access to RS of a social object is through the understanding of the ways individuals use to create, transform and interpret a problem related to their reality, as well as to know their thoughts, feelings, perceptions and experiences of shared life, of according to the social class to which they belong and the institutions to which they are linked. ${ }^{10}$

The representations are formed by systems of interpretations that lead the relations of the subjects with the world and with the others. $\mathrm{P}$ ortanto, study the RS about diabetes will provide an understanding of how it is treated the multidimensionality of this belongs to object by the group of people with diabetes mellitus, seeking to understand the representational field that includes information, images, beliefs, values, beliefs, cultural elements and ideological present in the discourse on the construct in question. ${ }^{11}$

According to Ribas et al. ${ }^{12}$ RS have a wide application in the field of health, especially in chronic conditions, as is the case of DM. In this theoretical model the psychosocial aspects involved are focused on the disease, which have been increasingly valorised them in clinical practice, especially for understanding the factors related to metabolic control and follow-up of patients with diabetes. This rate, this analis approach to people in full on your needs, considering them as biopsychosocial beings.

Therefore, para check what already exists in the literature on the subject in question has performed one search in the Capes Journal Portal, published in the last 11 years (January 01, 200 6-31 december 2017) from the combination of descriptors [social representations and diabetes] and their analogous terms in the English and Spanish languages; Inclusion criteria were adopted of papers discussing the social representations d diabetes.

With the search carried out, a total of 11 articles were found, which approached the following themes; RS about diabetes, diet, diabetic foot, body, about the health/illness relationship, RS about the care performed by professionals and family support. ${ }^{13-21}$ Des $t$ art, it is observed that despite the existence of supracita studies s, al available literature anchored in social psychological approach about diabetes developed by people with this condition are incipient, being more frequent in the area of nursing, with little emphasis on Psychology ( Rabbit et al \& Ethers P et al)

Thus, the formulation of shared knowledge about the DM may contribute as Coutinho et al., ${ }^{10}$ in and vid is INSTANCE of feelings, sensations, knowledge and beliefs about the disease, and to identify how social actors with diabetes diagnosis face the adversities, verifying what are the most significant aspects in the process of illness and treatment and what behaviours adopted in face of this problem. The TRS will enable a closer relationship with the subjectivity of individuals which will give grants for health promotion and prevention against chronic disease.

\section{Method}

It is a field research, descriptive, quantitative and qualitative, subsidized in a psycho - sociological approach. 31 people participated in the study aged between 34 to 76 years $(\mathrm{M}=55.68, \mathrm{SD}=11.6) \mathrm{d}$ which are the most female and RA (26) are married (18), who studied up $(\mathrm{M}=12.19 ; \mathrm{SD}=9.69)$ and who reported that they did not use insulin (16). The sample was non-probabilistic and for convenience. The inclusion criteria were: (i) to be 18 years or older; (ii) have a diagnosis of DM. The research was developed in an institution that maintains an agreement with the Unified Health System (SUS) and serves people with diabetes, located in the city of João Pessoa/PB.

To obtain the data, a Biosociodemographic Questionnaire and the Interview in Depth were used. The first instrument was used with the purpose of obtaining information about the profile of the participants, such as; age, sex, schooling, marital status, time of diagnosis and use of insulin. Made use of in depth interview preceded based on the following question: Please like that (a) you (a) speak everything you know about Diabetes Mellitus, including your feelings, feelings, and beliefs. This instrument allows the interviewee to freely on the subject proposed, without answers or conditions fixed by the researcher, which permit and further clarification on the inter relationship of the individual with the object of study, showing day experiences day, affective and existential issues, among others, elaborated on the basis of the common sense language, being these essential factors for the apprehension of RS. 22,23

The research was approved by the Research Ethics Committee of the Federal University of Paraíba-CEP/CCS, under protocol number 914.880. During its implementation, all the ethical conditions established by the National Health Council Commission, created by Resolution 466/2012 and with a constitution designated by Resolution 246/97, were respected.

After receipt of the favourable opinion, contact the board of chosen institution, where the researchers presented themselves with a letter containing the objectives and importance of the development of the research. The data were then collected individually, in the CAIS, where the participants were waiting for the consultations. All participants signed a Free and Informed Consent Form, as well as how they were informed of the voluntary nature and confidentiality regarding their identification. The number of participants was established according to the saturation criterion defined by Sá (1998). In accordance with this criterion, interviews may be closed at the time tomatic content begins to repeat itself. It should be noted that the total application time of the instruments was, on average, 35 minutes.

The 31 interviews were recorded and transcribed in full, giving rise to the corpus that was processed by the Alceste software (AnalyseLexicale para Contexted'a Ensemble de Segments de Text) developed by Reinsert in $1986 .{ }^{24}$

This program has the objective of performing a lexical analysis, through statistical procedures, carried out in four stages: (i) And tapa A, is responsible for the recognition of ICUs (Initial Context Units) that correspond to the number of interviews performed and separation of the text in fragments of similar sizes, which are the UCEs ( Elementary Context Units ); ( ii ) E tapa B-refers to the definitive division and grouping of UCEs from the similarity of words; ness is the step which is applied the method of Hierarchical Classification Descending (CHD); ( iii )E tapa C-presents the stable 
description of the UCEs classes and the dendrogram of the CHD that illustrates the relations between classes based on the characteristic vocabularies (lexical) and fixed variables. Is D-cutting, that the statistical significance of the words in the classes or $p$ is measured through the frequency (f) and the chi -square statistic $\left(\chi^{2}\right)$;(iv) E tapa D-responsible for the accomplishment of complementary calculations and the contextualization of the vocabulary typical of each class. ${ }^{24}$

The questionnaire data were processed by means of Statistical Package for Social Science, version 19.0 (SPSS-19.0), and analyzed from the descriptive statistics, observing the frequency, the percentage, the mean and standard deviation. It should be pointed out that in order to use the variables, age and treatment time in the Alceste software; these were grouped considering intervals of 10 years.

\section{Results and discussion}

Data from the interview and processed by the software Alceste origin plow one corpus consists of 31 ICU s, totaling 19. 246 occurrences, being 2. 509 different words. By reducing the vocabulary to its roots, we found 419 analyble radicals and 392 UCE s. The Correspondence Factorial Analysis (AFC) retained $77 \%$ of the total corpus ECUs, resulting in a factorial plane shown in Figure 1. This figure shows the layout of two axes, which reflect the content of the speech participants, is distributed in four zones or conglomerates, in a non-random way and corresponding to the specific forms of the classes. In the horizontal line, Axis 1 reveals the largest factor loads identified in Alceste, accounting for $41 \%$ of the total variance of the UCEs. In this axis, on the negative or left-hand side of Figure 1, the words agglutinated in Class 4 (bread, whole, rice, coffee, piece and avoid) stand out in the discourse of female participants over 21 diagnosis of diabetes (Figure 1).

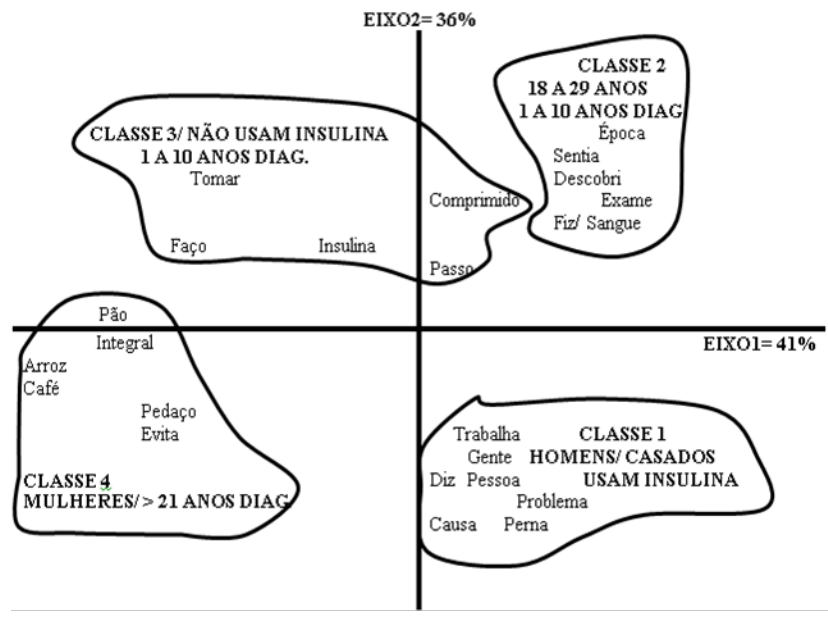

Figure I Factorial analysis of correspondence generated by ALCESTE $(n=$ $31)$.

In class 4 , the aspects related to the nutritional scope were observed, this group pointed to the description of the foods that make up the diet, schedules and quantities, as well as to elucidate homemade techniques to reduce the glucose of the organism, as illustrated in the following section. My diet is this morning my coffee is banana, a piece of papaya, I do not drink coffee, I drink a lot of juice with sweetener, I like to eat bread, but the doctor said that I cannot eat I had to eat one or two during the week and (...) everything as a whole cookie, rice, lean meat, vegetables, fruit I usually eat two, three, up to four times as the nutritionist informs (...) everything has to be a small and small quantity. ..) I drink water from okra already about six months ago.

On the other hand, the participants of this class also reported some foods that should not be ingested, as described in the UCEs: "There are many things that we cannot eat, so fat, sweet food, with lots of sugar, lots of juices, except with sweetener (...) as sometimes a small piece of cake ... not like everything in the middle, as an advanced medium."

In class 4 , diabetes was anchored in its nutritional aspects and objectified in the description of foods that make up the diet, schedules and quantities, and some homemade techniques to decrease the glucose of the organism. Moreover, in this class it was also possible to perceive the propagation as a determinant communication system of the content and form of the clarified objectifications. In this case the messages were produced and addressed to the members of the group, noting that some members of the group adopted attitudes to follow the diet to the detriment of the other participants, to maintain the posture of not doing the same. ${ }^{8,9}$

The amount of food was expressed using words such as "small piece", " medium " and "small quantity"; in this case, RS about diabetes was directed at the idea that people with this disease should eat a small amount of food. In the study of Ribas et al., ${ }^{12}$ for the participants of the research the concept of being well fed was related to the quantity (and not quality) of food consumed, inferring that eating sparingly or in moderation is not synonymous to be well fed.

This category of analysis refers to the understanding that hunger is satiated and formulated in cultural, social, economic and historical terms. Food consists of a basic human need, but it also constitutes a social act. How much, what, when, how, where and with whom one eats, other than food prescriptions and proscriptions, are factors determined by culture. In addition, the practices, classifications and representations of food demonstrate the status of an individual in a given society. In this sense, food can be interpreted as language, as a cultural text that translates worldviews, family, body, work and social relations (Menasche et al.).

Studying food through the referential of social representations means understanding that the person, in his social and cultural context, also chooses food to compose his food according to his subjective aspects, in the way he believes to be correct and in the way he translates the transmitted guidelines by pr health ofissionais. ${ }^{12}$

Foods that should be avoided are described as fatty or full of sugar, suggesting the urge to ingest these unhealthy foods. Coelho et al., ${ }^{25}$ recommend that people with DM should learn self-control and follow the instructions established by the health team, in order to establish and maintain treatment follow-up behaviours. The conduct of ingesting or restricting dietary foods can have many negative long-term implications, such as weight gain and, consequently, lack of disease control, although there are also reinforcing consequences in the short term that be effective in strengthening the behaviour of eating inappropriate food.

Finally, participants also reported using home-made techniques such as "okra water" to add to the treatment of diabetes. According to Barsaglini, ${ }^{18}$ it is common for people with diabetes to use coadjutant strategies to manage their treatment, such as home-made teas, natural medicines and prayers. Of course, the use of these alternative therapeutic resources does not exclude the use of the drugs. 
Regarding the description of the factorial plane, in the vertical axis, on the positive side, to the right, the words with the highest factorial loads grouped in Class 2 (time, felt, discovered, examination, done and blood) were placed, which emerged of patients aged 18 to 29 years and diagnosed between 1 and 10 years.

Class 2 refers to the lack of knowledge about diabetes, as well as the process of finding the disease, whether it was routine examinations or using a glucose meter (fingertip capillary glucose meter) in their homes. Another aspect evidenced was the onset of the disease due to a hereditary component, as can be observed in the following reports.

I used to hear a lot about diabetes, but I did not know what it was I did not take care of it, my mother was, but I did not care about the problem either... I discovered with the routine examination. I told you no, I have nothing ... my family history was very painful, father, mother, uncles, aunts, grandparents, all died of diabetes and cancer.

Participants were found to have anchored diabetes in factors related to their diagnosis. The most prominent elucidations were: lack of knowledge about diabetes; discovery of the disease through examinations, or directed to hereditary factors, visualized after the death of relatives.

According to Rodrigues et al., ${ }^{15}$ the lack of knowledge about diabetes may be related to low schooling. The present study corroborates this information, since of the 31 interviewees, 28 studied until elementary school. The few years of study can also collaborate with the non adherence to the therapeutic plan because of the difficulty to read and understand the prescription, thus increasing the health risks. In addition, low level of schooling may limit access to information, probably because of impairment of reading, writing and speaking skills, as well as understanding the complex mechanisms of disease and treatment. ${ }^{15}$

The discovery of diabetes revealed through periodic medical examinations points to its natural evidence process according to Barsaglini. ${ }^{18}$ Identification may also occur by surprise, surprising the person due to the absence of objective or subjective manifestations, although validated initially by laboratory confirmation..$^{18}$ In this study, this accidental finding was seen through the use of the glucometer at home.

Participants also evidenced the hereditary issue, although there is an increase in the number of cases, especially due to the current habits of the population, marked by sedentary lifestyle and inadequate diet (Whiting et al. 2011). Perhaps the lack of knowledge about the main factors that trigger diabetes could contribute to a better level of prevention since the disease is caused by environmental and hereditary factors.

The emotional factor was also a component that excelled in this class. The people who most contributed their accounts to this class described that being diabetic is synonymous with sadness, crying, suffering, bad thoughts, depression and death, have claimed that it is a disease that kills slowly. As exemplified in the following UCEs.

I became a sad person and I face a lot of depression after I discovered diabetes (...) being diabetic is very sad, I feel like crying is a disease that kills a little (...) in my head a lot of bad happens to I am death and suffering.

The emotional character that the illness brings to these people was also evident in this class, through the objectifications: "sadness, crying, suffering, bad thoughts, depression and death." It is noteworthy that it was through these expressions that the participants gave materiality to the emotional reality experienced after the diagnosis of diabetes.

According to Peres et al., ${ }^{21}$ present chronic health condition involves several changes in the habits and daily life of individuals, which can be extremely difficult and difficult to accept. Receiving the diagnosis of a chronic disease such as diabetes arouses various feelings, emotional reactions and fantasies that need to be known and understood by health professionals. The objections highlighted by the participants of this study are in line with the study by Peres et al., ${ }^{21}$ when investigating RS about diabetes in women living in this condition, who reported that the diagnosis was marked with sadness, anger, revolt and shock.

The emotional instability that may have been triggered at the time of the news of the disease may persist later, interfering in the metabolic control of these people. For Santos et al., ${ }^{17}$ these negative feelings, when crystallized, can predispose to the formation of depressive symptoms, such as low self-esteem, discouragement, hopelessness, low expectation of the future, loss of vital tone and interest in daily activities - including those considered important for maintenance of good metabolic control.

Regarding Axis 2, in the vertical line of Figure 1, two groups of words stand out, explaining $36 \%$ of the total variance of the UCEs. It emerged semantic two fields: one in the upper plane, with contributions from PA participants who were not taking insulin and diagnosed between 1 and 10, d Class 3 (take pill, I, insulin, and I); and, by contrast, in the lower plane, the words from the male participants, married and using insulin, are categorized in Class 1 (work, people, says, person, problem, cause and leg).

With regard to class 3 , located in the upper plane, the participants expressed both favourable and unfavourable reaction towards compliance with the treatment, with neglect always accompanied by a justification, according to the following reports:

I diet and walk, I eat every three hours, taking better care of myself and taking the medication (...) control my insulin, I take four times a day, two doses in the morning, before lunch and dinner and the tablet. I do not dieting, but I'm taking medicine, before lunch and after dinner ... the doctor told me to walk had some time I did, but I stopped already way from the bus stop to my house ... when I travel do not take medicine, (...) sometimes I diet, I think every diabetic is undisciplined, do not do any physical activity, I start to walk and suddenly stop.

In this class, diabetes was anchored in biomedical knowledge about treatment and, therefore, one can identify the visibility of this knowledge through the expressions: diet, medication and physical activity. Consonant Tavares et al., ${ }^{7}$ patients with diabetes should reconcile the triad mentioned above, since it is this structure of treatment.

In relation to physical activity, some participants reported that they walk, either formally as an organized and disciplined activity, offered by public and private establishments, or informally practicing a similar activity, for example, walking from the bus stop to the residence. According to Barsaglini ${ }^{18}$ although the participation of people with diabetes in organized physical activities is not so common, many of these people guarantee to practice similar activities in the domestic space itself: cleaning, washing, cooking, going and walking back to work, walking with animals or looking after family members. 
Although the participants reported whether or not to follow diet and physical activity, medication intake was unanimous. In this conjecture, medication seems to be the basis of treatment, due to its legitimacy and the recognition of its results in the control of diabetes. ${ }^{18}$ Because it is a long-term disease, this, in turn, requires commitment to therapy, requiring the person's coping ability throughout his life. Sometimes the commitment to follow or the desire to stop treatment, even momentarily, is always present in the diabetic person's daily life. $^{21}$

In relation to lasses 1 , found in the bottom plane of the horizontal axis is observed content $\mathrm{s}$ related to the concept of diabetes as well as notions of responsibility, obligations and conscience towards compliance with the treatment, according to the following reports from participants.

I'm not very knowledgeable about diabetes (...) it's a complicated , silent disease (...) I see that it does not take care of me (...) treatment have to have a lot of discipline, Difficult life of the person who has diabetes (...) be aware that tomorrow The can lose a finger, a leg or arm, blind the we step on the brake of these things and tries to walk, run, ride (...) I cannot complain about follow-up, no shortage orientation is with the person who did not call me, do not point the doctor is doing her job What is diabetes?, it just depends on me.

As the stories of the participants with the profile already mentioned it is observed that diabetes concept in the sense common if features analogue to concept of knowledge scholar, one turn what it is considered $\mathrm{u}$ bad disease complex and silent, which requires discipline in your treatment, although in Most of the time, the people no have the awareness of following it, unless what $\mathrm{P}$ erect am what in the future can experience the implications per she imposed $\mathrm{s}$. The consequence more noticeable to men married and that they make use of insulin it was the loss of fear of some member of the body, namely: finger, leg, arm, still, in to develop vision problems.

Given the above referring to class 1 , if you can realize that diabetes was anchored at perception of responsibilities and obligations facing the risk in develop the consequences arising from the disease. It is categorization was related to knowledge Medical both facing the instance more severe disease, which are the amputations, so as for your internship Home, for it is a disease silent, being recognized only after the worsening of its symptomatology. Brazil $(2013)^{26}$ assesses what half the population who have diabetes do not know their symptoms. For Stuhler (2012) the difficulties resulting from diabetes can be related to complications disabling, which compromise the productivity, quality of life and survival, leading impacts psychosocial at people's lives.

Those participants have evidenced, also that the larger responsible for the good progress of treatment and changes in your lifestyle are they same as one turn what receive the guidelines professionals. According Ribas et al., ${ }^{12}$ some participants recognize the guidelines medical as a resource positive for coping with the disease, taking your portion of liability through the no adherence of measures what could favouring a better control metabolic. In that case comprises-the assumption of a posture resigned due to the difficulty to break the movement inertial and instituting the changes needed we habits of life.

Those patients are the demand and special attention from health professionals, aiming instruct them to move to a position of greater engagement in habits healthy. As well, since what exist a representation Positive service, you need to draw advantage of this benefit and work to strengthen the belief these patients in your ability to overcome barriers what obstruct access at changes longed. ${ }^{12}$

\section{Conclusion}

Based on main objective of identify the representations social elaborated per people what have diabetes about the disease and treatment, the present study allowed the rescue of the knowledge of the sense common, with evidence of different conceptions, feelings, attributes and images what permeated the whole construction of knowledge and as made possible understand as these people perceive That problematic and how guide your ducts against the disease contributing to the overall understanding of DM.

Diabetes mellitus was represented as disease silent, complex and multifaceted, whose diagnosis is evidenced delayed due the absence of symptoms Specific and ignorance of the syndrome. Also, It was found that through narratives susceptibility of diabetics they are favourable to comorbidities so much physical how much psychoemotional, as is the case of symptomatology depressive, according to the stories guided by the nature of humor negative; " sadness, crying, suffering, bad thoughts and death ".

How much to treatment, it was found that this one was guided at triad: medicines, diet and activities physical, despite the lack of rigor in the engagement exercises. So broad, it has been observed that lack of commitment to the therapy It generates a sense of autoculpabilização mainly When the diabetics they see themselves at imminence of serious consequences, such as the loss of limbs or vision.

In view of the above, RS of the disease for this group was organized according to the knowledge bio psychosocial. The results point to the importance of a approach interdisciplinary what focus or support psycho-sociological facing strategizing what can be adopted front to control and care of diabetes, which glimpse mainly larger clarifications about the causes and consequences of is $\mathrm{s}$ the disease as well as incentive practices The $r$ MPLEMENTATION activities physics.

It implies it is that such practices consider in the influence of the particularity of the social and cultural context experienced by people with diabetes, aiming avoid a posture merely submitting to Principles biom doctors. It is important to what that group can be made to reflect about acceptance of the disease and of their attempts behavioural front to treatment. Per finally, it suggests-if the development of studies facing the process of resilience and wellness subjective, aiming to focus as that belonging group overcomes adversity originating of this context.

\section{Acknowledgements}

None.

\section{Conflict of interest}

Author declares that there are no conflicts of interest.

\section{References}

1. Stuhler GD. Representations social and adhesion to treatment of type 2 diabetes mellitus 2. Graduate Program in Social Psychology, Federal University from Santa Catarina, Florianópolis. 2012. 
2. http://www.diabetes.org/are-you-at-risk/prediabetes/?\%201oc $\% 20=\% 20$ superfooter

3. Rodríguez SA, Meneses L, Toboso RPQ. HI diabetes mellitus. Medicine. 2012;11(17):995-1002.

4. Oliveira JEP, Vencio S. Corporate guidelines Brazilian diabetes association: 2013-2014/ society Brazilian diabetes association. São Paulo: AC Pharmaceuticals. 2014.

5. Whiting DR, Guariguata R, Weil C, et al. Diabetes Atlas: Global estimates of the prevalence of diabetes for 2011 and 2030. Diabetes Res Clin Pract. 2011;3(94):311-321.

6. http://www.idf.org

7. Tavares BC, Barreto FA, Lodetti MR, et al. Resilience of people with diabetes mellitus. Text nursing context. 2011;20( 4):751-757.

8. Coutinh MPL, Saraiva ERA. Theory of representations social policies. In NT Alves et al. (Orgs.), Psychology: reflections for teaching, research and extension. João Pessoa, PB: Publisher University. 2013;73-114.

9. Moscovici S. Representations social: Investigations in social psychology 7th ed. Petrópolis, RJ: Voices. 2012.

10. Coutinho. MPL Depression Children and representations social. 2nd ed. João Pessoa: Publisher University/UFPB. 2005.

11. Saraiva, ERA Violence against the elderly: approximations and distances between the speech of the elderly and the discourse of the media printed. Doctoral thesis, Graduate Program in Social Psychology, Federal University of Paraíba, João Pessoa. 2010.

12. Ribas CR, Santos MA, Zanetti ACG. Representations social of patients with diabetes mellitus on the care per health professionals. Magazine Psycho. 2013; 44(1):139-149.

13. Costa FG, Coutinho, MPL Representations social in the context of diabetes mellitus. Psychology in Study. 2016;21(1):175-185.

14. Mantovani AM, Fegonesi CEPT, Pelai EB. Study comparison of representations social on diabetes mellitus and foot diabetic. Notebooks in Cheers Public. 2013;29(12):2427-2435.

15. Rodrigues FFL, Santos MA, Teixeira CRS, et al. Relationship between knowledge, attitude, schooling and time of illness in individuals with diabetes mellitus. Minutes Paulista of Nursing. 2012;25( 2):284-290.
16. Ribas CRP, Santos MA, Zanetti. Representations Social food under optic of people with diabetes mellitus. Interamerican Journal of Psychology. 2011;45(2):255-262.

17. Santos MA, Alves RCP, Oliveira VA, et al. Representations social of people with diabetes about the perceived family support in relationship to treatment. Revista da Escola de Enfermagem da USP. 2011;45 (3):651658.

18. Barsaglini RA. Representations social and experience with diabetes: a focus socio-anthropological. Rio de Janeiro: Publisher Fiocruz. 2011.

19. Coelho MS, Silva DMGV, Padilha. MIS Representations Social foot diabetic for people with diabetes mellitus type 2. Magazine USP School of Nursing. 2009;43(1):65-71.

20. Péres DS, F ranco LJ, Santos MAD. Behaviour to feed in women with type 2 diabetes. Rev Saude Publica. 2006;40(2):310-317.

21. Péres DS, Franco LJ, Santos MA, et al. Representations social women diabetic , layered popular in Relationship to process health-disease. Latin American Journal of Nursing. 2008;16( 3).

22. Vieira KFL. Sexuality and quality of life of the elderly: challenges contemporary and repercussions psychosocial. D thesis granted, Graduate Program in Social Psychology, Federal University of Paraíba, João Pessoa. 2012.

23. Min ayo M. The challenge of knowledge: research qualitative in Cheers. Rio de Janeiro: Abrasco. 2007.

24. Saraiva ERA, Coutinho MPL, Miranda RS. The use of Alceste Software and the development of the lexical world in documentary research. In MPL Coutinho \& ERA Saraiva, editors. Search Methods in social psychology: perspectives qualitative and quantitative. João Pessoa, PB: Publisher University. 2011:67-94.

25. Coelho CR, Amaral VLAR. Analysis if a contingency with diabetes mellitus type 2: case study. Psico-USF. 2008;13(2):243-251.

26. Ministry of Health. Department of Health Care. Department of Attention Basic. Strategies for care of the person with illness chronic: diabetes mellitus/ministry of health care department of health, department of attention basic. Brasilia: ministry of Health, Brazil. 2013:160. 